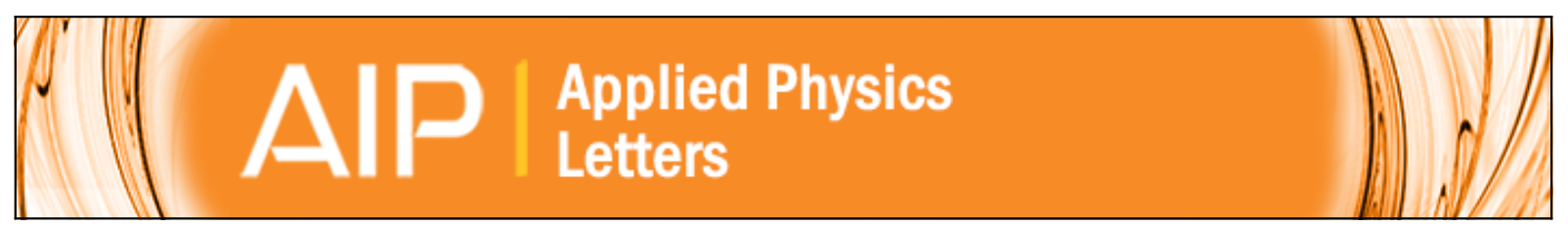

\title{
Imaging regenerating bone tissue based on neural networks applied to micro- diffraction measurements
}

G. Campi, G. Pezzotti, M. Fratini, A. Ricci, M. Burghammer, R. Cancedda, M. Mastrogiacomo, I. Bukreeva, and A. Cedola

Citation: Applied Physics Letters 103, 253703 (2013); doi: 10.1063/1.4852056

View online: http://dx.doi.org/10.1063/1.4852056

View Table of Contents: http://scitation.aip.org/content/aip/journal/apl/103/25?ver=pdfcov

Published by the AIP Publishing

\section{Articles you may be interested in}

The fatigue life prediction of aluminium alloy using genetic algorithm and neural network

AIP Conf. Proc. 1554, 174 (2013); 10.1063/1.4820313

Strategies to associate memories by unsupervised learning in neural networks

AIP Conf. Proc. 1510, 255 (2013); 10.1063/1.4776533

Online monitoring and error detection of real-time tumor displacement prediction accuracy using control limits on respiratory surrogate statistics

Med. Phys. 39, 2042 (2012); 10.1118/1.3676690

Design and validation of a novel bioreactor principle to combine online micro-computed tomography monitoring and mechanical loading in bone tissue engineering

Rev. Sci. Instrum. 81, 014303 (2010); 10.1063/1.3284787

An artificial neural network for predicting the incidence of radiation pneumonitis

Med. Phys. 32, 318 (2005); 10.1118/1.1835611

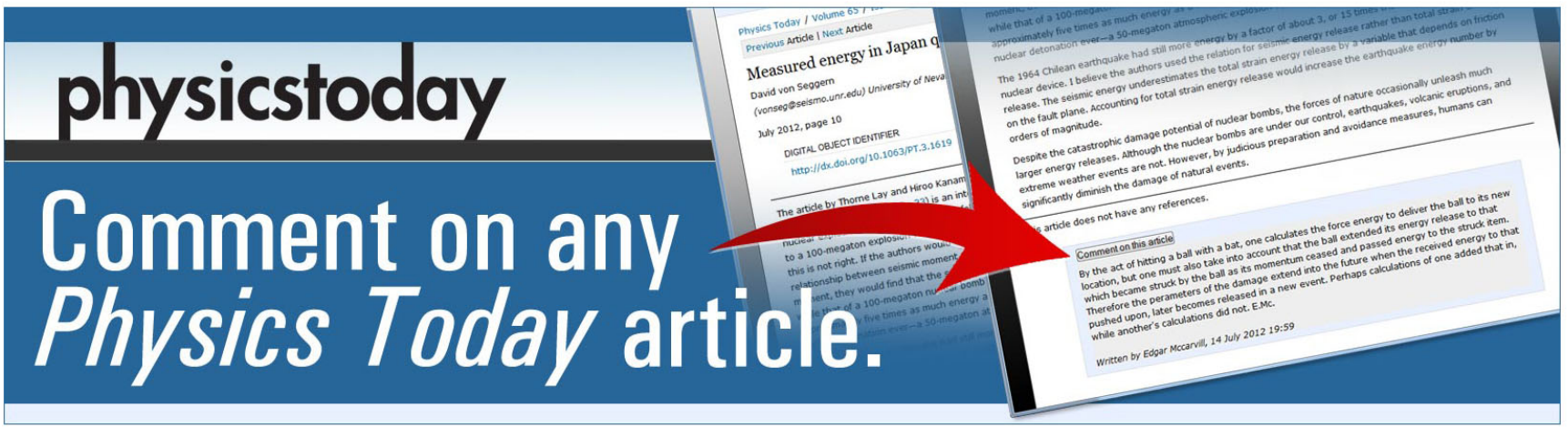




\title{
Imaging regenerating bone tissue based on neural networks applied to micro-diffraction measurements
}

\author{
G. Campi, ${ }^{1}$ G. Pezzotti, ${ }^{1}$ M. Fratini, ${ }^{2}$ A. Ricci, ${ }^{3}$ M. Burghammer,${ }^{4}$ R. Cancedda,${ }^{5}$ \\ M. Mastrogiacomo, ${ }^{5}$ I. Bukreeva, ${ }^{6}$ and A. Cedola ${ }^{6, a)}$ \\ ${ }^{1}$ Institute of Crystallography, CNR, via Salaria Km 29.300, I-00015, Monterotondo Roma, Italy \\ ${ }^{2}$ Centro Fermi -Museo Storico della Fisica e Centro Studi e Ricerche "Enrico Fermi", Roma, Italy \\ ${ }^{3}$ Deutsches Elektronen-Synchrotron DESY, Notkestraße 85, D-22607 Hamburg, Germany \\ ${ }^{4}$ European Synchrotron Radiation Facility, B. P. 220, F-38043 Grenoble Cedex, France \\ ${ }^{5}$ Istituto Nazionale per la Ricerca sul Cancro, and Dipartimento di Medicina Sperimentale dell' Università di \\ Genova \& AUO San Martino Istituto Nazionale per la Ricerca sul Cancro, Largo R. Benzi 10, 16132, \\ Genova, Italy \\ ${ }^{6}$ Institute for Chemical and Physical Process, CNR, clo Physics Dep. at Sapienza University, P-le A. Moro 5, \\ 00185, Roma, Italy
}

(Received 28 June 2013; accepted 3 December 2013; published online 19 December 2013)

\begin{abstract}
We monitored bone regeneration in a tissue engineering approach. To visualize and understand the structural evolution, the samples have been measured by X-ray micro-diffraction. We find that bone tissue regeneration proceeds through a multi-step mechanism, each step providing a specific diffraction signal. The large amount of data have been classified according to their structure and associated to the process they came from combining Neural Networks algorithms with least square pattern analysis. In this way, we obtain spatial maps of the different components of the tissues visualizing the complex kinetic at the base of the bone regeneration. (C) 2013 AIP Publishing LLC. [http://dx.doi.org/10.1063/1.4852056]
\end{abstract}

Heterogeneous media are typically composed of multiple phases coexisting on micrometric scale. This characteristic makes quite difficult to model their structural features with conventional experimental approaches, requiring high spatial resolution probes. X-ray micro diffraction ( $\mu \mathrm{XRD})$ is a powerful tool allowing to carry out fundamental structural information on multiple length scale onto small areas, combining wide range of momentum transfer with smallness of probed domains. This wide range in reciprocal space allows to measure momentum transfer from Small Angle X-ray Scattering (SAXS) to Wide Angle X-ray Scattering ranges (WAXS); in this way, the structural properties can be investigated simultaneously from nano to atomic arrangement. ${ }^{1,2}$ At the same time, the smallness of probed domains, obtained by the small $\mathrm{X}$ ray beam size, is particularly appropriate in the study of heterogeneous samples, where different phases coexist on (sub)micrometric areas. Recently, the heterogeneity in matter has been attracting more and more attention because of its role in functional materials in several fields ranging from material science ${ }^{3-5}$ to biology, ${ }^{6-9}$ and medicine. ${ }^{10}$ This is particularly true in the tissue engineering and in biomedical field where tissues are naturally complex and show multiscale heterogeneity. ${ }^{11,12}$ One of the most typical examples is given by the bone tissue whose formation occurs via dynamic interactions between supra-molecular assemblies of bio-macromolecules producing mixtures of different organic and inorganic phases. ${ }^{13,14}$ The organic phase consists of matrix proteins, mostly collagen (C) type I, with some minor non-collagenous proteins and minor amounts of lipids and osteogenic factors (e.g., bone morphogenetic proteins). ${ }^{15}$ The inorganic phase is composed by calcium phosphates in

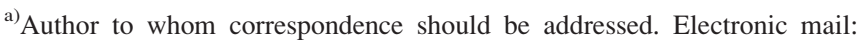
alessia.cedola@cnr.it. Tel.: +390641522260.
}

both crystalline, Hydroxyapatite (HA), and amorphous (ACP) state. ${ }^{16}$ Thus, monitoring what happens in the region where organic-mineral interactions occurs, becomes crucial and requires probes able to catch tissue structural properties with high spatial resolution.

In this framework, we investigated the biomineralization process in an ectopic bone formation mouse model using $\mu \mathrm{XRD}$. In our model, ex vivo expanded bone marrow mesenchymal stem cells are seeded onto a porous ceramic scaffold and implanted subcutaneously in the mouse. ${ }^{17}$ After the established implantation time the scaffold is removed from the host animal and the newly formed bone analyzed. We monitored the early stage in the bone tissue development scanning the sample with a micrometric sized beam at different distances from the scaffold (SC), corresponding to different mineralization stage. ${ }^{13} \mathrm{We}$ found mainly two different type of tissues: the new bone tissue (B) formed by HA nanoparticle crystallization and the connective soft tissue (ST) formed through the vascularization process and filling the scaffold pores.

The tissues spatial distribution corresponded to a temporal monitoring of the bone growth and the mineralization process. Thus, in order to image the structural evolution of bone tissue formation, we needed to locate the different tissues, by classifying each measured diffraction pattern according to its provenance tissue. Neural networks are typically used to perform complex functions, such as pattern recognition, identification, classification, and control systems, ${ }^{18,19}$ aiming to mimic the biological nervous systems by connection of based elements, neurons. A neural network can be trained to perform a particular function by adjusting the values of the connections, weights, so that a particular input leads to a specific target output. Since each diffraction pattern shows specific features from the different specific 
tissue, here we apply neural networks at basic level, as a signal recognition tool, to spatially classify and associate each measured diffraction pattern to its proper type tissue that is SC or B or ST. Moreover, for each type of tissue we distinguished different phases in different reciprocal space $q$-range, corresponding to organic, $\mathrm{C}$, inorganic $\mathrm{HA}$ and $\mathrm{ACP},{ }^{13}$ quantified by a least square approach. Finally, we composed spatial maps of these phases, in the different tissues, evidencing interesting features of mammalian bone tissue formation.

Bone Marrow Stromal Cells (BMSC) were obtained from iliac crest marrow aspirates from healthy adult sheep (Italian Biellese strain). All donor animals had reached sexual maturity, animal care and treatment is conducted in conformity with institutional guidelines in compliance with national and international laws and policies (European Economic Community (EEC) 1987; Guide for the Care and Use of Laboratory Animals 1996; Kon 2000). BMSC cultures were performed as previously described. ${ }^{20}$ Osteogenic properties of the BMSC were evaluated by an "in vivo" assay in an immuno-deficient mice model. ${ }^{21}$ First passage cells expanded in standard medium were trypsinized, resuspended in a fibrinogen solution (Tissuecol; Baxter, Italia) to a final concentration of $62.5 \times 10^{6} \mathrm{cells} / \mathrm{ml}$ and loaded onto highly porous ceramic scaffolds $(100 \%$ hydroxyapatite cubes, $3 \times 3 \times 3 \mathrm{~mm}^{3}$; FinCeramica, Faenza, Italy); an appropriate volume of $20 \mu \mathrm{l}$ of Thrombin was added to ignite the enzymatic cleavage that originates a fibrin clot around and within the ceramic, entrapping the cells. After 4 weeks the samples were harvested, washed in Phosphate Buffered Saline (PBS) three times and fixed in paraformahaldehyde (4\% in PBS) for $2-3 \mathrm{~h}$ at $4{ }^{\circ} \mathrm{C}$. Additional washes in PBS removed the residual fixative. Than the sample was dehydrated in ethanol at increasing concentration, embedded in methylmetacrilate and transversally cut using a diamond saw (Gillings- Hamco, Hamco Machines, Inc., Rochester, N.Y., U.S.A) in serial sections $(\approx 100 \mu$ m thick) for the $\mathrm{X}$-ray measurements.

The X-ray micro-diffraction measurements on the thin sections of the bone tissue, extracted after 4 weeks, were carried out at the ID13 beamline of the European Synchrotron Radiation Facility (ESRF) in Grenoble. The double-crystal monochromator and a Kirkpatrick-Baez mirror system supply a beam size of $1 \times 1 \mu \mathrm{m}$ with a wavelength of $0.976 \AA$. The $y-z$ scanning micro-diffraction setup used a piezoscanning stage with an accuracy of $0.1 \mu \mathrm{m}$. The scanning step used in both y and z direction was $5 \mu \mathrm{m}$. Diffraction patterns were recorded in transmission by a MARCCD detector with a typical acquisition time of $5 \mathrm{~s}$. 2D diffraction patterns have been radially integrated to provide $1 \mathrm{D}$ profiles of intensity vs. transfer moment, $q$.

Several samples of different scaffolds were implanted in different animals for 4 weeks. Figure 1 shows the section of one of the analyzed samples. Close to the scaffold (white region) we have the newly formed bone, while the soft tissue (black region) fills the pores. Since the bone starts growing to the scaffold interface and proceeds towards the pore center, different distances from the scaffold correspond to different mineralization stages. ${ }^{13}$ The origin and development of $\mathrm{B}$ and ST tissues have been studied by $\mu \mathrm{XRD}$, scanning the sample from the center of pore towards the scaffold, covering a quite large momentum transfer, $q=4 \pi \sin (\theta) / \lambda$ from
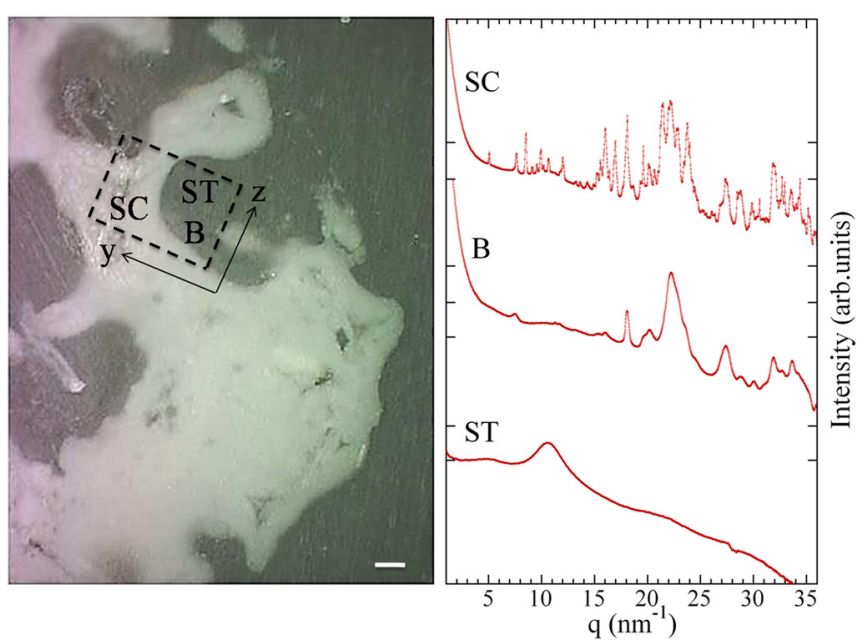

FIG. 1. Section of a measured sample; the dashed rectangle represents the area on which the microdiffraction measurements have been done, using a beam size of $1 \times 1 \mu \mathrm{m}$ and a scanning step of $5 \mu \mathrm{m}$ along $\mathrm{y}$ and $\mathrm{z}$ directions. The unit bar corresponds to 100 microns. The different diffraction signals, corresponding to the SC, B, and ST tissue type are reported on the right panel.

$1.0 \mathrm{~nm}^{-1}$ to $36 \mathrm{~nm}^{-1}$. The resulting profiles, recorded at the different positions $(\mathrm{y}, \mathrm{z})$, and characterizing the different tissues, namely SC, HA, and ST, are shown in the right panel of Figure 1. The SC tissue shows XRD patterns with sharp peaks, due to micrometric size of HA crystallites. In bone tissue, B, the peaks become broadened, as typically occurs in nanocrystal, because of the crystallization of the HA nanoparticles in collagen interstitial space. Finally, the soft tissue, $\mathrm{ST}$, filling the pore, is due to the vascularization providing osteoblasts cells releasing the organic collagenous and non-collagenous connective matrix.

The first classification for mapping these three basic tissues has been carried out using neural networks. Our data sets consisted of $\mathrm{N}$ x M XRD patterns as input vectors, while for each type tissue, $\mathrm{T}$, we considered a specific two-element target vectors, composed by 1 and 0 . The value 1 means the $\mathrm{X}$-ray pattern corresponding to the correct tissue, while 0 is associated to the other type tissues. Then, we used a pattern recognition feed-forward back-propagation network ${ }^{22}$ with tan-sigmoid transfer functions in both the hidden layer and the output layer. We used 20 neurons in the hidden layer and one neuron in the output layer. The pattern recognition network used the default Scaled Conjugate Gradient Backpropagation algorithm ${ }^{23}$ for training. The training application was performed on inputs profiles constituting about the $1 \%$ of the total $\mathrm{M} \times \mathrm{N}$ profiles; these selected input profiles were randomly divided into three sets: $70 \%$ are used for training, $15 \%$ are used to validate that the network was generalizing and to stop training before overfitting, while the last $15 \%$ were used as a completely independent test of network generalization. The training stopped when the validation error increased for six iterations. The network outputs resulted to be extremely good in all three type of tissues, achieving always correct responses larger than the $95 \%$ both in the validation and in the testing. Scheme of neural network response analysis is shown in Figure 2. After the training, the network was simulated on all the $\mathrm{M} \times \mathrm{N}$ diffraction patterns. For each tissue type these $\mathrm{M} \times \mathrm{N}$ inputs profiles give an output matrix of $\mathrm{M} \times \mathrm{N}$ real-values comprised 


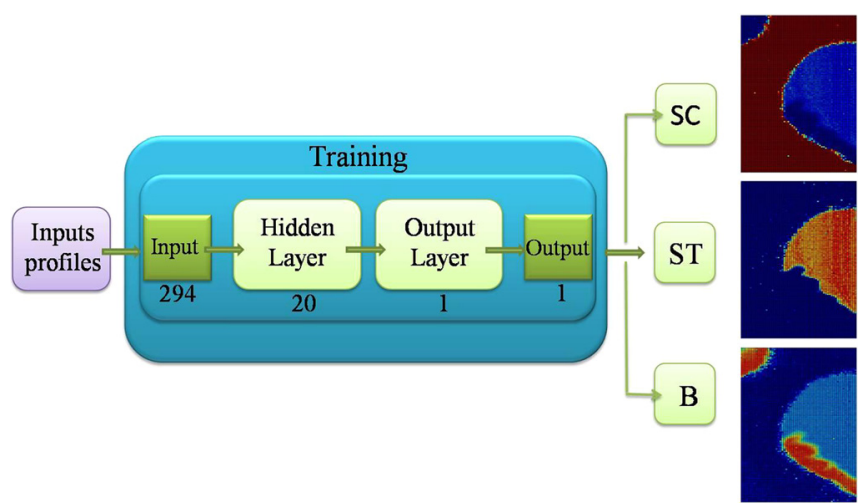

FIG. 2. Layout of neural networks algorithm used to classify the different tissue type, SC, ST, and B. The inputs consisted of $\mathrm{M} \times \mathrm{N}$ vectors of 294 elements, with $\mathrm{M}=121$ and $\mathrm{N}=61$. Each input vector was extracted from the whole diffraction profile in the WAXS q-region $\left(18<\mathrm{q}<28 \mathrm{~nm}^{-1}\right)$, where differences between the various tissues, are easily identifiable (see Fig. 1).

between 0 and 1 . We considered correct responses, matrix elements higher than 0.5 . As results, each XRD pattern was classified accordingly with its tissue typology, allowing us to define a mask $M_{T}$ for each tissue type, $T$ : $M_{S C}$ for Scaffold, $\mathrm{M}_{\mathrm{B}}$ for Bone, and $\mathrm{M}_{\mathrm{ST}}$ for Soft Tissue.

In each region corresponding to the defined tissue, the diffracted intensity allowed us to appreciate the different phases, corresponding to different $q$-ranges. In particular the following phases $(\mathrm{P})$ have been evidenced: (i) HA nanocrystals formed at early and later stage, monitored both in the SAXS range as HA nanoparticle formation and in the WAXS range as crystallization of the same HA nanoparticles; $^{24}$ (ii) the collagen, $\mathrm{C}$, corresponding to the peak at $\mathrm{q}=5.6 \mathrm{~nm}^{-1},{ }^{13,25,26}$ (iii) the ACP individuated by the broad peaks at $\mathrm{q}=21.1 \mathrm{~nm}^{-1}$ as found in the synthetic counterpart. ${ }^{27}$ In order to build kinetic maps of these phases in the different tissues, T, (namely SC, B, and ST) the contribute of each phases, $\mathrm{P}$, (namely HA, C, and ACP) has been quantified as

$$
I_{T}(P)=\int_{q\left(P_{i n}\right)}^{q\left(P_{f i n}\right)}\left[I_{E X P}(q)-I_{B K G}(q)\right] d q * M_{T},
$$

calculated for each specific $q$-range. The $\mathrm{C}$ and ACP peaks have been modeled as Gaussians around $q$ values of
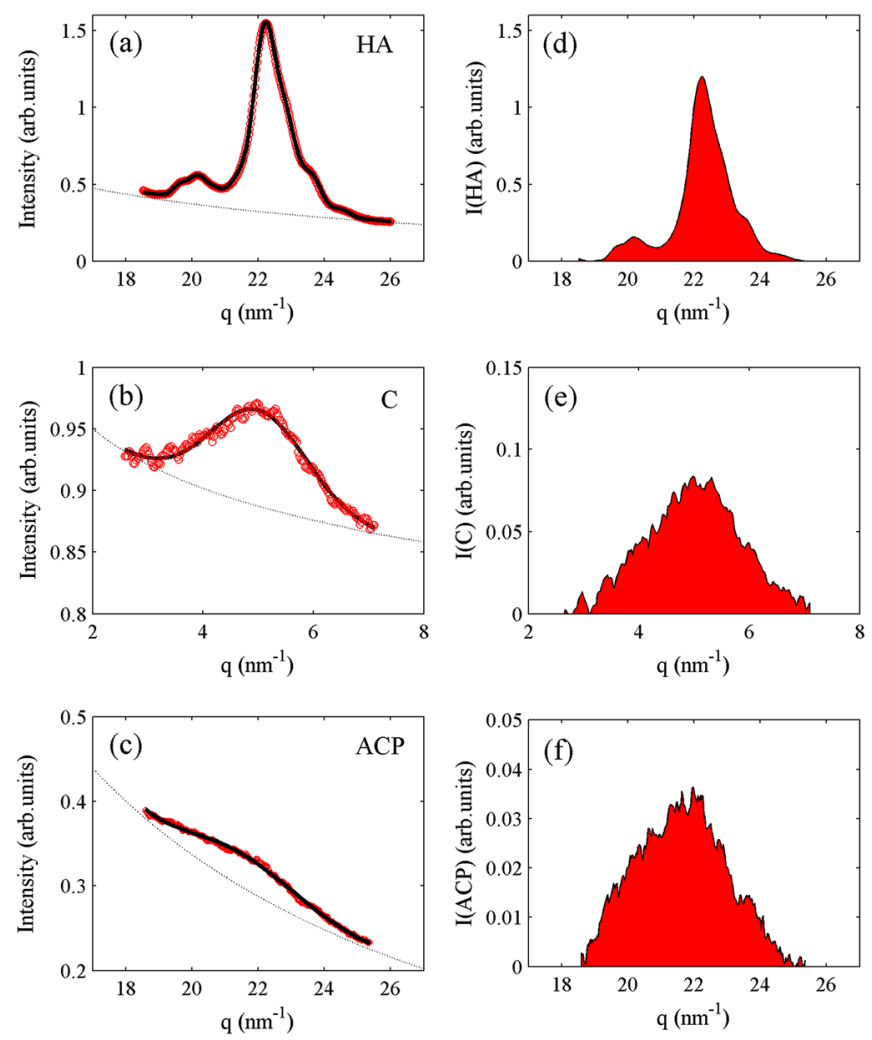

FIG. 3. Fits of $X$ ray patterns in specific q-range corresponding to (a) HA nanocrystals, (b) collagen, C, and (c) ACP. In (d), (e), and (f) we show the area of signals in (a), (b), (c) with $\mathrm{I}_{\mathrm{BKG}}$ subtraction, and corresponding to the $\mathrm{I}(\mathrm{HA}), \mathrm{I}(\mathrm{C})$ and $\mathrm{I}(\mathrm{ACP})$ quantities calculated by (1).

$5.6 \mathrm{~nm}^{-1}$ and $21.1 \mathrm{~nm}^{-1}$, respectively, with a power law background $\mathrm{I}_{\mathrm{BKG}}$. The HA nanocrystals have been modeled by a Rietveld crystallographic structure with cell parameters $\mathrm{a}=9.4162, \mathrm{~b}=9.4162$, and $\mathrm{c}=6.8791 \AA$. We used the least square method in order to estimate the peaks amplitude and width, and the background, $\mathrm{I}_{\mathrm{BKG}}$, as illustrated in Figure 3. At this point the $\mathrm{I}(\mathrm{P})$ in (1) can be calculated for each type tissue, obtaining the kinetic maps of bone tissue regeneration, as shown in Figure 4. In Fig. 4(a), the intensity I(HA) corresponding to the newly formed bone tissue is illustrated demonstrating to be originated by the formation of HA nanocrystals nucleating at the $\mathrm{B} / \mathrm{ST}$ interface and growing towards the SC. In Figs. 4(b) and 4(c) we show the distribution of collagen, $\mathrm{C}$, and ACP, respectively, in the ST and B tissues. We observed how the $\mathrm{C}$ and ACP distribution is
HA

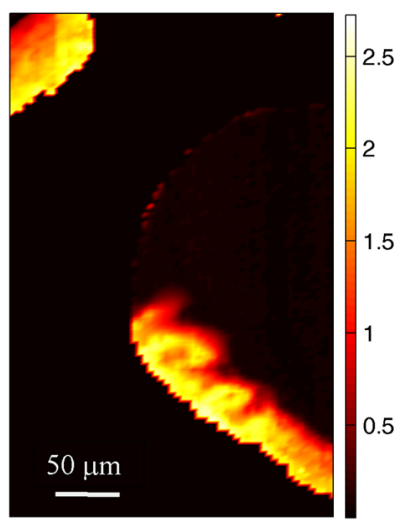

Collagen

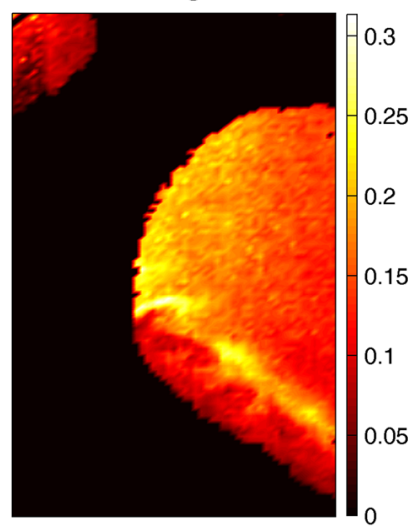

ACP

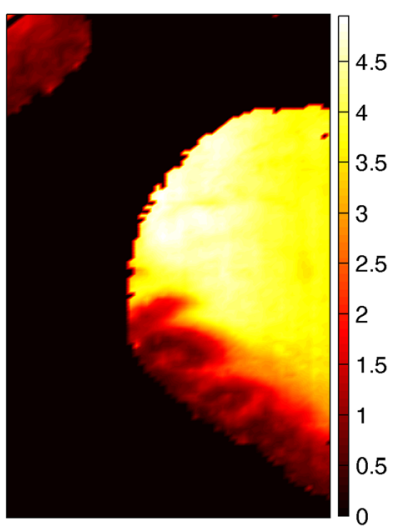

FIG. 4. Spatial distribution of the intensity $\mathrm{I}(\mathrm{HA}), \mathrm{I}(\mathrm{C})$, and $\mathrm{I}(\mathrm{ACP})$ in the $\mathrm{B}$ and ST tissues. The scaffold (black region) has been masked. 

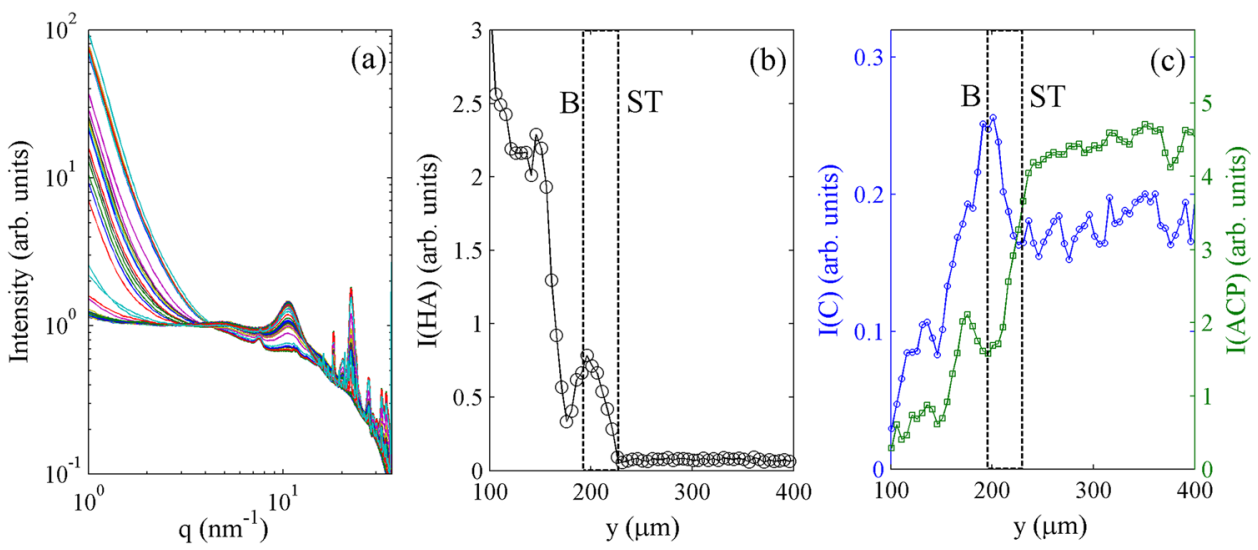

FIG. 5. (a) Diffraction profiles measured scanning the sample along $\mathrm{z}$, crossing the $\mathrm{B} / \mathrm{ST}$ interface indicated by the dashed rectangle in (b) and (c); (b) I(HA) intensity of WAXS signal, (c) I(C) and I(ACP) intensities calculated by (1). The B/ST interface is defined as the layer where (i) the WAXS signal starts to increase and (ii) where the $\mathrm{C}$ and ACP signal are anticorrelated. quite correlated in the ST tissue, while it becomes anticorrelated at the B/ST interface where the ACP takes to decrease, transforming in crystalline bone tissue. On the contrary, at the same interface the collagen goes thickening. In Figure 5, we show the intensities $\mathrm{I}(\mathrm{HA}), \mathrm{I}(\mathrm{C}), \mathrm{I}(\mathrm{ACP})$ along a typical vertical profile crossing the $\mathrm{B} / \mathrm{ST}$ interface in the $\mathrm{y}$ direction. The described behavior is clearly visible in the B/ST interface, about $30 \mu \mathrm{m}$ thick, indicated by the dashed rectangle: we can visualize the ACP-Bone tissue transformation assisted by the enhancement of collagen packing, at the organic-inorganic interface. Further analysis, involving fibres collagen orientation mapping and signal modelling on the whole $q$-range will be needed to get deeper insight on the biomineralization process, here visualized.

In summary, structural heterogeneity in biological tissues can be mapped, thanks to highly spatially resolved probes. Scanning micro X-ray diffraction allows getting basic structural information joining large momentum transfer in reciprocal space onto small areas in real space. Combining neural-network methods for classifying the type tissue with least square fitting methods for characterizing the various structural phases in each tissue, we achieved the doubled handled result (i) to compose clear spatial maps of structural phases involved in the tissue regeneration and (ii) to get additional insights on its underlying complex mechanism. We hope that this study could contribute to set means of imaging heterogeneous biological tissues as well as monitoring tissue structure evolution during various medical treatments.

${ }^{1}$ P. Fratzl, H. S. Gupta, E. P. Paschalis, and P. Roschger, J. Mater. Chem. 14, 2115 (2004).

${ }^{2}$ A. Guagliardi, A. Cedola, C. Giannini, M. Ladisa, A. Cervellino, A. Sorrentino, S. Lagomarsino, R. Cancedda, and M. Mastrogiacomo, Biomaterials 31, 8289-8298 (2010).

${ }^{3}$ N. Tamura, R. Spolenak, B. C. Valek, A. Manceau, M. Meier Chang, R. S. Celestre, A. A. MacDowell, H. A. Padmore, and J. R. Patel, Rev. Sci. Instrum. 73, 1369-1372 (2002).

${ }^{4}$ M. Fratini, N. Poccia, A. Ricci, G. Campi, M. Burghammer, G. Aeppli, and A. Bianconi, Nature 466, 841-844 (2010).

${ }^{5}$ G. Campi, A. Ricci, N. Poccia, L. Barba, G. Arrighetti, M. Burghammer, A. S. Caporale, and A. Bianconi, Phys. Rev. B 87, 014517 (2013).
${ }^{6}$ A. Cedola, V. Stanic, M. Burghammer, S. Lagomarsino, F. Rustichelli, R. Giardino, N. Aldini Nicoli, M. Fini, V. Komlev, and S. Di Fonzo, Phys. Med. Biol. 48(3), 37-48 (2003).

${ }^{7}$ H. Iwamoto, K. Inoue, T. Fujisawa, and N. Yagi, J. Synchrotron Radiat. 12, 479-483 (2005).

${ }^{8}$ O. P. Posnansky and N. J. Shah, J. Biol. Phys. 34, 551-567 (2008).

${ }^{9}$ G. Ciasca, M. Papi, M. Chiarpotto, M. Rodio, G. Campi, C. Rossi, P. De Sole, and A. Bianconi, Appl. Phys. Lett. 100, 073703 (2012).

${ }^{10}$ C. S. Kamma-Lorger, S. Hayes, C. Boote, M. Burghammer, M. E. Boulton, and K. M. Meek, Mol Vis. 15, 378-385 (2009).

${ }^{11}$ S. Lin, Y -S Lee, R. Narayan, and H. Shin, in Proceedings of 2007 Industrial Engineering Research (IERC) Conference, Nashville, TN, 19-23 May 2007, pp 281-286.

${ }^{12}$ S. Lin, Y.-S. Lee, and R. Narayan, Int. J. of Mechatronics and Manufacturing Systems 1(1), $43-67$ (2008).

${ }^{13}$ G. Campi, A. Ricci, A. Guagliardi, C. Giannini, S. Lagomarsino, R. Cancedda, M. Mastrogiacomo, and A. Cedola, Acta Biomater. 8(9), 3411-3418 (2012).

${ }^{14}$ M. J. Olszta, X. Cheng, S. SooJee, R. Kumar, Yi -Yeoun Kim, M. J. Kaufman, E. P. Douglas, and L. B. Gower, Mater. Sci. Eng. R. 58(3-5), 77 (2007).

${ }^{15}$ B. Ganss, R. H. Kim, and J. Sodek, "Bone sialoprotein," Crit. Rev. Oral Biol. Med. 10(1), 79-98 (1999).

${ }^{16}$ S. V. Dorozhkin, Int. J. Mater. Chem. 2(1), 19-46 (2012).

${ }^{17}$ R. Quarto, M. Mastrogiacomo, R. Cancedda, S. M. Kutepov, V. Mukhachev, A. Lavroukov, E. Kon, and M. Marcacci, N. Engl. J. Med. 344, 385 (2001).

${ }^{18}$ D. Reby, S. Lek, I. Dimopoulos, J. Joachim, J. Lauga, and S. Aulagnier, Behav. Processes 40, 35-43 (1997).

${ }^{19}$ G. Pezzotti, J. Valencia, and N. Londono, IEEE Lat. Am. Trans. 10(4), 1967-1972 (2012).

${ }^{20}$ E. Kon, A. Muraglia, A. Corsi, P. Bianco, M. Marcacci, I. Martin, A. Boyde, I. Ruspantini, P. Chistolini, M. Rocca, R. Giardino, R. Cancedda, and R. Quarto, J. Biomed. Mater. Res. 49, 328 (2000).

${ }^{21}$ A. Muraglia, I. Martin, R. Cancedda, and R. Quarto, Bone 22, 131S-134S (1998).

${ }^{22}$ C. M. Bishop, Pattern Recognition and Machine Learning (Springer, New York, 2006), Vol. 1.

${ }^{23}$ E. M. Johansson, F. U. Dowla, and D. M. Goodman, Int. J. Neural Syst. 2(4), 291-301 (1991).

${ }^{24}$ Th. Leventouri, C. E. Bunaciu, and V. Perdikatsis, Biomaterials 24, 4205-4211 (2003).

${ }^{25}$ A. Cedola, M. Mastrogiacomo, M. Burghammer, V. Komlev, P. Giannoni, R. Cancedda, F. Rustichelli, A. Favia, and S. Lagomarsino, Phys. Med. Biol. 51, N109-N116 (2006).

${ }^{26}$ A. Cedola, M. Mastrogiacomo, S. Lagomarsino, R. Cancedda, C. Giannini, A. Guagliardi, M. Ladisa, M. Burghammer, F. Rustichelli, and V. Komlev, Spectrochim. Acta, Part B 62(6), 642-647 (2007).

${ }^{27}$ J. Mahamid, B. Aichmayer, E. Shimoni, R. Ziblat, C. Li, S. Siegel, O. Paris, P. Fratzl, S. Weiner, and L. Addadi, Proc. Natl. Acad. Sci. U.S.A. 107(14), 6316-6321 (2010). 